\title{
A Simulation of the Reception of Automatic Dependent Surveillance-Broadcast Signals in Low Earth Orbit
}

\author{
Richard Van Der Pryt and Ron Vincent \\ Royal Military College of Canada, P.O. Box 17000, Station Forces, Kingston, ON, Canada K7K 7B4 \\ Correspondence should be addressed to Ron Vincent; ron.vincent@rmc.ca
}

Received 4 March 2015; Accepted 13 July 2015

Academic Editor: Gonzalo Seco-Granados

Copyright (C) 2015 R. Van Der Pryt and R. Vincent. This is an open access article distributed under the Creative Commons Attribution License, which permits unrestricted use, distribution, and reproduction in any medium, provided the original work is properly cited.

\begin{abstract}
Automatic Dependent Surveillance-Broadcast (ADS-B) is an air traffic surveillance technology in which aircraft transmit position and identification. The development of space-based ADS-B will allow precise control of aircraft in areas that are not covered by radar, such as oceanic regions and high latitudes. The Royal Military College of Canada has developed a spaceborne ADS-B receiver scheduled to fly on the Canadian Advanced Nanospace eXperiment-7 (CanX-7) satellite. The payload is planned to collect data over the North Atlantic region, which will then be compared to truth data provided by air traffic services. A model was created to determine power levels arriving at the satellite to provide confidence in the ADS-B receiver and antenna proposed for CanX-7. The model takes into account neutral atmosphere and ionospheric effects, aircraft-satellite geometry, and antenna radiation patterns. A simulation was run by inserting real aircraft data from the North Atlantic Track System into the model and placing the satellite at altitudes of 400,600 , and $800 \mathrm{~km}$. Results of the simulation indicate that power received at the satellite, ranging between $-98.5 \mathrm{dBm}$ and $-103 \mathrm{dBm}$ for the selected altitudes, will be sufficient to successfully conduct the mission.
\end{abstract}

\section{Introduction}

Automatic Dependent Surveillance-Broadcast (ADS-B) is an air traffic surveillance technology in which aircraft routinely transmit identification and position information during flight. The system uses a navigational reference aboard the aircraft, often a global positioning system (GPS) receiver, to determine the aircraft's position. The ADS-B information is transmitted in a coded message at frequent, random intervals throughout the flight. These signals can be received by other aircraft or by ground stations for relay to air traffic services (ATS). The use of satellite navigation systems and the frequent update cycle allow ADS-B to provide more accurate and timely surveillance information than conventional surveillance radars. This enables aircraft to send surveillance and collision avoidance information to each other.

The ADS-B information is transmitted over three bearers: Mode S (or $1090 \mathrm{MHz}$ ) extended squitter (ES), universal access transceiver (UAT) on $978 \mathrm{MHz}$, and very high frequency data link (VDL Mode 4) in the $130 \mathrm{MHz}$ range. In 2002 the American Federal Aviation Administration announced that $1090 \mathrm{MHz}$ would be used in the next generation ATS for air carrier and private/commercial operators of high performance aircraft. The ES ADS-B message is a 120-bit transmission that contains aircraft identification, position, velocity, and status. The message is broadcast with a period that ranges randomly between 0.4 and 0.6 seconds to prevent aircraft from having synchronized transmissions. Required transmitter power for the ADS-B transmission varies between $75 \mathrm{~W}$ and $500 \mathrm{~W}$, depending on the aircraft category [1]. The vertically polarized ADS-B signals alternate between top- and bottom-mounted quarter-wave monopole antennas.

ADS-B has come into use in recent years in several regions, providing surveillance information to ATS where radar coverage is not available or improving the performance of the surveillance system in cooperation with radar. Canada's ATS provider, NAV CANADA, has implemented ADS-B surveillance in the Hudson Bay and the Labrador/South Greenland regions with other regions to come in the near 
future. Other airspace authorities, notably in Europe, the United States, and Australia, plan to implement ADS-B surveillance in the coming years.

Although the implementation of ADS-B is growing, ground stations cannot be installed in mid-ocean and are difficult to maintain in the Arctic, leaving a coverage gap for oceanic and high latitude airspace. An orbital surveillance system has the potential to expand the capabilities of the current terrestrial surveillance system, providing traffic monitoring capability in areas where radars and ground stations do not exist or where their installation is impractical or prohibitively expensive. The introduction of precise and reliable surveillance in remote and oceanic airspace would allow an improvement over existing air traffic procedures, which use standardized routes and large inter-aircraft spacing to provide aircraft separation in the absence of surveillance data. With surveillance information available, aircraft on long flights could take more direct routes, saving time and fuel, thereby reducing engine emissions. The reduced aircraft spacing requirements would also allow more effective use of increasingly crowded airspace, such as the North Atlantic region. These efforts are topical with the announcement of ADS-B receivers being used as secondary payloads on the Iridium Next constellation [2]. Experimental ADS-B payloads were launched on the European Space Agency Proba-V and GOMX-1 satellites in 2013 and have shown that ADS-B transmissions can be retrieved in low Earth orbit (LEO).

The Royal Military College of Canada (RMCC) has been involved in ADS-B research since 2009, conducting successful high altitude balloon missions and publishing research in the field [3-6]. As a result of this work, RMCC developed an ADS-B receiver that is scheduled to fly as a technology demonstrator on the Canadian Advanced Nanospace eXperiment-7 (CanX-7) nanosatellite. To assess the likelihood of success of the mission, a model was created to determine power levels arriving at the satellite. The model takes into account neutral atmosphere and ionospheric effects, aircraft-satellite geometry, and antenna radiation patterns. A simulation was run by inserting real aircraft data from the North Atlantic Track System (NATS) into the model and placing the satellite in low Earth orbit (LEO).

The paper is organized as follows. Section 2 describes the CanX-7 mission and the ADS-B payload. Section 3 details the atmospheric effects that were considered in the creation of the ADS-B signal propagation model. Section 4 discusses the model, while Section 5 describes the results of the simulation. Section 6 contains conclusions and future work.

\section{CanX-7 ADS-B Payload}

2.1. Satellite and Mission Description. CanX-7 is being developed by the University of Toronto Institute of Aerospace Studies (UTIAS) as a technical demonstrator of a deployable drag sail for passive spacecraft deorbiting [7]. The secondary payload is the RMCC ADS-B receiver. CanX-7 is a $3 \mathrm{U}$ format cubesat that measures $10 \times 10 \times 34 \mathrm{~cm}$ with a mass less than $4 \mathrm{~kg}$. The deorbiting technology is a lightweight, modular, deployable drag sail providing $5 \mathrm{~m}^{2}$ of sail area following deployment [7]. Scheduled for launch in 2016, the CanX-7 mission concept is to wait six months after successful on-orbit checkout to allow operation of the ADS-B payload before deploying the sail. Orbital parameters, including altitude and inclination, are currently unknown since a launch has not yet been brokered. It is assumed that the orbital altitude will fall between 400 and $800 \mathrm{~km}$ with an inclination that will allow coverage of the North Atlantic airspace.

2.2. ADS-B Payload and Antenna. The ADS-B receiver/ decoder, Radarcape, was developed from the "Mode S Beast" receiver. The Radarcape payload was chosen following a successful RMCC high altitude balloon mission in 2012 . Radarcape is supported by a single board computer called the Beaglebone rather than relying on an external computer to record data. The Beaglebone is an ARM Cortex A8 processor mounted on a development board that has expansion connectors, on-chip Ethernet, a micro-SD card, and a USB host port that typically runs the Ångström Linux distribution. The receiving antenna is a circularly polarized patch antenna. The half-beamwidth angle is $55^{\circ}$ with overall gain of $-2.35 \mathrm{~dB}$ at $1090 \mathrm{MHz}$. The antenna bandwidth, which is still to be determined, will fall between 4 and $29 \mathrm{MHz}$, resulting in a minimum detectable signal between $-98.5 \mathrm{dBm}$ and $-103 \mathrm{dBm}$, respectively.

2.3. ADS-B Mission Profile. ADS-B data will be collected over the North Atlantic region in the Gander and Shanwick Oceanic Control Areas (OCAs). NAV CANADA provides air traffic services in the Gander OCA and maintains archival data for the Shanwick OCA. This region was chosen since it is relatively quiet with respect to $1090 \mathrm{MHz}$ transmissions and truth data will be available to assess the performance of the payload. ADS-B information will be stored onboard the satellite then subsequently downlinked at UTIAS. Data analysis, including signal strength, lost transmissions, and signal collision probability will be conducted at RMCC.

\section{Atmospheric Effects}

The Earth's atmosphere can be divided into ionized and neutral regions. ADS-B signals will travel through both these regions as it propagates from aircraft to satellite. Ionospheric effects are examined followed by neutral atmosphere effects in relation to ADS-B signals.

3.1. Ionospheric Signal Propagation Analysis. The ionosphere is a region of ionized plasma caused by solar activity typically between 50 and $2000 \mathrm{~km}$ altitude. The frequency $(f)$ dependence of ionospheric effects is generally $1 / f^{2}$, which means the greatest influence is at low frequencies. Ippolito [8] suggests a propagation analysis procedure for satellite links operating below $3 \mathrm{GHz}$ that is summarized by the flowchart in Figure 1.

3.1.1. Total Electron Content (TEC). A number of ionospheric effects are related to TEC, so it is important to know 


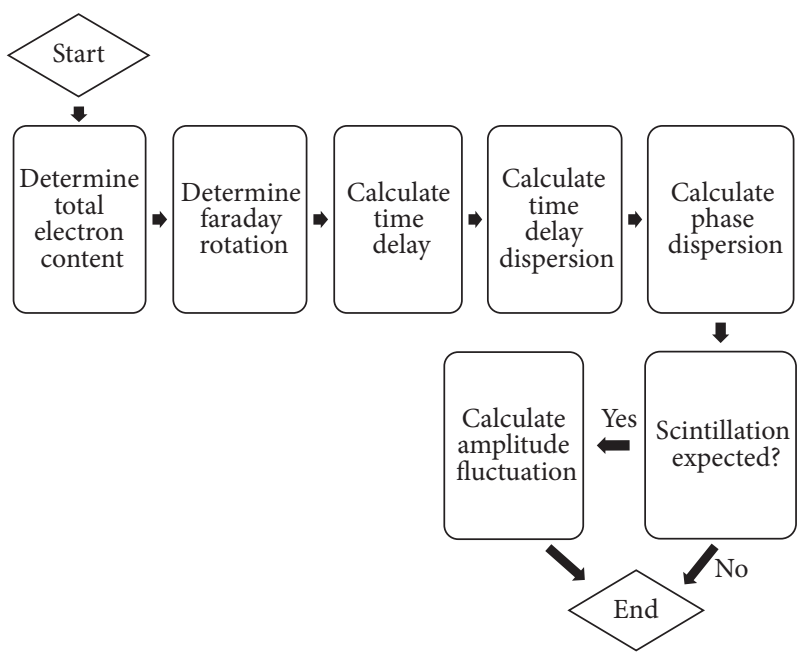

FIGURE 1: Analysis procedure for satellite links below $3 \mathrm{GHz}$ (abridged) [8].

its magnitude before ionospheric effects can be estimated quantitatively. Slant total electron content (STEC) is the measurement of electron concentration along the propagation path given by [9]

$$
\operatorname{STEC} \int_{s} n_{e}(s)(d s),
$$

where $n_{e}$ is the electron concentration in electron $/ \mathrm{m}^{3}$ and $s$ is the propagation path in $\mathrm{m}$.

For satellite predictions, the TEC value typically used is for a zenith path having a cross section of $1 \mathrm{~m}^{2}$. This value is referred to as vertical total electron content (VTEC) and varies between $1 \times 10^{16}$ and $1 \times 10^{18}$ electrons $/ \mathrm{m}^{2}$. The number of electrons along a path is normally referred to in TEC units (TECU) where 1 TECU $=1 \times 10^{16}$ electrons. Conversion between STEC and VTEC uses a model of the ionosphere where all the free electrons are assumed to be concentrated in a single infinitely thin layer at an altitude $H$ above the surface with an Earth radius of $R_{E}$ as shown in Figure 2. VTEC is then estimated at each ionospheric piercing point (IPP) according to

$$
\frac{\text { STEC }}{\mathrm{VTEC}}=\frac{1}{\cos z^{\prime}},
$$

where

$$
z^{\prime}=\sin ^{-1}\left(\frac{R_{E}}{R_{E}+H} \sin z\right)
$$

3.1.2. Faraday Rotation. Faraday rotation is the rotation of the sense of polarization as an electromagnetic wave passes through a magnetic field in a transparent dielectric. This apparent rotation is caused by the different propagation speeds of left-hand and right-hand circular polarized waves. As a linearly polarized wave is a combination of two identical amplitude circular polarized waves of opposite handedness and phase shifted by $\pi$ radians, the effect of the relative phase shift is a rotation of the wave's linear polarization.

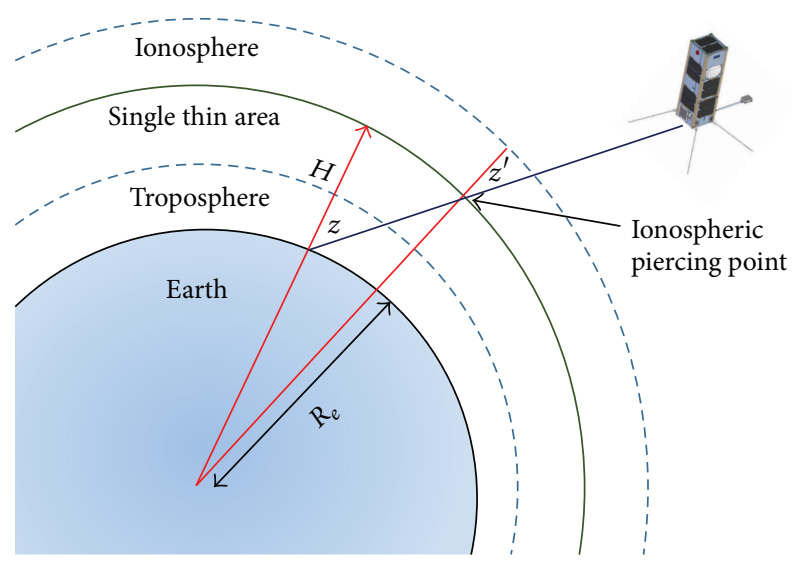

FIGURE 2: Single thin layer ionosphere model [9].

The magnitude of the rotation, $\varphi$, is given by [10]

$$
\varphi=\frac{2.36 \times 10^{4}}{f^{2}} B \cos \theta_{B} \mathrm{TEC},
$$

where $\varphi$ is the angle of rotation in radians, $f$ is frequency in $\mathrm{Hz}$, TEC is total electron content in electrons $/ \mathrm{m}^{2}, B$ is the Earth's magnetic field in $\mathrm{Wb} / \mathrm{m}^{2}$, and $\theta_{B}$ is the angle between the magnetic field and the direction of propagation. The magnitude of Faraday rotation for an ADS-B signal over the North Atlantic reigon to a LEO satellite is about $12.5^{\circ}$ using representative values for total magnetic field strength of 55,000 nT and a TEC of 20 TECU.

3.1.3. Time Delay and Time Delay Dispersion. Charged particles in the atmosphere slow the propagation of radio signals. Time delay is referenced to propagation in a vacuum and is represented by [10]

$$
\Delta t=\frac{1.345 \times 10^{-7}}{f^{2}} \text { TEC. }
$$

Time delay dispersion is the rate of change of time delay with frequency and is determined by differentiating (5)

$$
\Delta t=\frac{-2.69 \times 10^{-7}}{f^{3}} \text { TEC. }
$$

If a radio signal has a large bandwidth (BW), propagation delay will vary between the upper and lower frequencies, and dispersion will result. Equation (7) modifies (6) to calculate the time delay dispersion directly by including the signal pulse length, $\tau$,

$$
|\delta t|=\frac{2.69 \times 10^{-7}}{\tau f^{3}} \mathrm{TEC},
$$

where

$$
\left|\frac{1}{d f}\right|=\mathrm{BW}=\frac{1}{\tau} .
$$

For an ADS-B signal with a signal pulse length of $0.5 \mu \mathrm{s}$, a centre frequency of $1090 \mathrm{MHz}$, and a TEC value of $20 \mathrm{TECU}$, the time delay is $26 \mathrm{~ns}$ and the time delay dispersion is $83 \mathrm{ps}$. 
3.1.4. Phase Dispersion. Phase advance, $\Phi$, is the change in phase caused by the time delay described in (5) and is represented as [8]

$$
\Phi=\frac{2 \pi f}{c} \Delta t
$$

where $c$ is the speed of light. The rate of change of phase angle with frequency is called phase dispersion and is the derivative of phase advance [8]. Differentiating $\Phi$ with respect to frequency and incorporating signal pulse length from the relationship in (8) gives

$$
|\delta \Phi|=\frac{8.44 \times 10^{-7}}{\tau f^{2}} \text { TEC. }
$$

Using the same parameters of frequency and TEC from the previous examples, the phase dispersion is $16.3^{\circ}$ for the ADSB signal.

3.1.5. Scintillation and Absorption. Ionospheric scintillation results from rapid variations in local electron density that cause changes in refraction. Some regions focus the radio wave and others cause divergence resulting in varying signal levels. The International Telecommunication UnionRadiocommunication Sector (ITU-R) has developed an empirical method to estimate the effects of scintillation [11]; however the model is admittedly inaccurate [8]. Scintillation tends to a maximum near the geomagnetic equator at equinox during high sunspot activity [11].

Absorption in the ionosphere occurs as a result of increased electron concentration during high solar activity. The two most common types are auroral absorption and polar cap absorption (PCA). Auroral absorption occurs near the center of a visual aurora in the night portion of the polar region and has an average duration of 30 minutes. PCA normally occurs at the peak of the sunspot cycle and events typically have durations that last up to a few days and are detectable over the sunlit portion of the polar region. Auroral absorption and PCA are normally significant for frequencies below $100 \mathrm{MHz}$ and are not a factor for ADS-B signals.

3.2. Neutral Atmosphere Effects. Neutral atmosphere effects can be subdivided into clear air effects and particulate effects. Clear air effects include refraction, gaseous absorption, and tropospheric scintillation, while particulate effects include scattering and absorption.

3.2.1. Refraction. Refraction is the change in direction of an electromagnetic wave as it passes through media where the wave velocity varies. The ratio of the velocity in the media compared to the velocity of an electromagnetic wave in vacuum is called the refractive index, $n$. The combined dry and wet refractive index in the lower atmosphere (up to $50 \mathrm{~km}$ altitude) is given by [10]

$$
n=1+\left[\frac{77.6}{T} \cdot\left(P+\frac{4810 e}{T}\right) \times 10^{-6}\right],
$$

where $T$ is the absolute temperature in Kelvin and $e$ is the partial pressure of water vapor in $\mathrm{mb}$.
3.2.2. Gaseous Absorption. Nonsymmetrical molecules, or polar molecules, in the atmosphere tend to align themselves with an electric field. Changes to the electric field induce absorptive losses at resonant frequencies caused by the electric dipole. Water vapour is the main contributor to this kind of absorption. Oxygen, while nonpolar, has a permanent magnetic moment and also causes resonant absorption, although the mechanism is different from that of water vapour. Other polar molecules have an effect, but their contribution is small compared to those of water and oxygen below $70 \mathrm{GHz}$ [10]. Absorption of electromagnetic radiation due to water and oxygen is calculated by determining the specific attenuation coefficient, $\gamma$, in units of $\mathrm{dB} / \mathrm{km}$ for a given frequency along the path length. The attenuation coefficient for oxygen for $f<57 \mathrm{GHz}$ is [10]

$$
\begin{aligned}
\gamma_{o} & =\left[7.19 \times 10^{-3}+\frac{6.09}{f^{2}+0.227}\right. \\
& \left.+\frac{4.81}{\left(f^{2}-57\right)^{2}+1.50}\right] f^{2} \times 10^{-3} \mathrm{db} / \mathrm{km},
\end{aligned}
$$

while the attenuation coefficient for water vapor for $f<$ $350 \mathrm{GHz}$ is [10]

$$
\begin{aligned}
\gamma_{w} & =\left[0.050+0.0021 \rho+\frac{3.6}{(f-22.2)^{2}+8.5}\right. \\
& \left.+\frac{10.6}{(f-183.3)^{2}+9.0}+\frac{8.9}{(f-325.4)^{2}+26.3}\right] f^{2} \rho \\
& \times 10^{-4} \mathrm{db} / \mathrm{km} .
\end{aligned}
$$

For an ADS-B signal $\gamma_{o}=5.169 \times 10^{-3} \mathrm{~dB} / \mathrm{km}$ and $\gamma_{w}=$ $6.462 \times 10^{-3} \mathrm{~dB} / \mathrm{km}$.

Specific attenuation values should be integrated along the propagation path to account for variances in pressure and density with height. A simpler method that achieves this goal is through the use of equivalent heights, in which the contribution of the constituents is assumed to be zero. The equivalent height, $h_{o}$, for oxygen is $6 \mathrm{~km}$ for $f<57 \mathrm{GHz}[10]$. The equivalent height for water, $h_{w}$, for $f>350 \mathrm{GHz}$ is [10]

$$
\begin{aligned}
h_{w} & =h_{w 0}\left(1+\frac{3.0}{(f-22.2)^{2}+5}+\frac{5.0}{(f-183.3)^{2}+6}\right. \\
& \left.+\frac{2.5}{(f-325.4)^{2}+4}\right) \mathrm{km},
\end{aligned}
$$

where $h_{w 0}=1.6 \mathrm{~km}$ for clear weather and $2.1 \mathrm{~km}$ in rain. Total zenith attenuation, $A_{z}$, becomes

$$
A_{z}=h_{0} \gamma_{0}+h_{w} \gamma_{w} \mathrm{~dB} .
$$

If the elevation angle $\theta \geq 10^{\circ}$, the cosecant law can be used to calculate the one way absorption, $A_{a}$, for an emitter at sea level

$$
A_{a}=\frac{A_{z}}{\sin \theta} \mathrm{dB}
$$


When considering the altitude of an aircraft in kilometers, $h_{a c}$, (16) is modified to

$$
A_{a}=\frac{\gamma_{o} h_{o} e^{-h_{a c} / h_{o}}+\gamma_{w} h_{w} e^{-h_{a c} / h_{w}}}{\sin \theta} \mathrm{dB} .
$$

Similarly, for elevation angles between $0^{\circ}$ and $10^{\circ}$,

$$
\begin{aligned}
A_{a} & =\frac{\sqrt{a_{e}}}{\cos \theta}\left[\gamma_{o} \sqrt{h_{o}} e^{-h_{a c} h_{o}} \mathbf{F}\left(\tan \theta \sqrt{\frac{a_{e}}{h_{o}}}\right)\right. \\
& \left.+\gamma_{w} \sqrt{h_{w}} e^{-h_{a c} h_{w}} \mathbf{F}\left(\tan \theta \sqrt{\frac{a_{e}}{h_{w}}}\right)\right] \mathrm{dB},
\end{aligned}
$$

where $a_{e}$ is the effective earth radius and the function $\mathbf{F}(x)$ is

$$
\mathbf{F}(x)=\frac{1}{0.661 x+0.339 \sqrt{x^{2}+5.51}} .
$$

The effective Earth radius corrects for atmospheric refraction and permits the assumption of straight ray paths, in which

$$
A_{e}=k R_{E},
$$

where $R_{e}$ is the Earth radius and $k$ is the radius coefficient. The value of $k$ ranges from 1.33 at very low elevation angles to 1.0 at zenith.

3.2.3. Tropospheric Scintillation. Tropospheric scintillation arises from the fact that the atmosphere is not a perfect series of stratified layers where the refractive index varies continuously with changing altitude but rather is subject to vertical mixing and turbulence. These small-scale fluctuations in refractive index vary the amplitude and phase of a wave propagating through them in the same manner as ionospheric scintillation. One difference between tropospheric and ionospheric scintillation is that tropospheric scintillation increases with increasing frequency. At $1 \mathrm{GHz}$, tropospheric scintillation is not normally considered in ground to satellite propagation for elevation angles $>1^{\circ}[10]$.

3.2.4. Particulate Effects. The contributors to particulatebased attenuation of radio waves in decreasing order are raindrops, cloud, and fog followed by sand and dust. If the size of the particle is significantly smaller than the radio wavelength, Rayleigh scattering is predominant but this changes to Mie scattering as the ratio of particle size to wavelength increases. The transition frequency from Rayleigh to Mie scattering for rain occurs at about $3 \mathrm{GHz}$, so ADS-B signals are within the Rayleigh scattering region. It is difficult to theoretically calculate the effects of scattering and absorption of rain separately, so the ITU-R has developed an empirical powerlaw relationship for rain specific attenuation, $\gamma_{R}$, which combines both effects [12]:

$$
\gamma_{R}=k R^{\alpha} \mathrm{db} / \mathrm{km},
$$

where $k=0.000308$ and $\alpha=0.8592$ for vertical polarization at $1 \mathrm{GHz}$. For a heavy rainfall rate of $100 \mathrm{~mm} / \mathrm{hr}, \gamma_{R}=$ $0.00161 \mathrm{~dB} / \mathrm{km}$.
Similar to the method used to calculate equivalent height for water vapour in (14), equivalent rain height for latitude > $23^{\circ} \mathrm{N}$ is given by [13]

$$
h_{R}=5-0.075(\phi-23) .
$$

For the middle of the North Atlantic OCAs with a latitude of $55^{\circ} \mathrm{N}, h_{R}=2.6 \mathrm{~km}$. Similar to (15), the zenith attenuation is given by

$$
A_{R z}=h_{R} \gamma_{\mathrm{Z}} \mathrm{dB} .
$$

Using the cosecant law the one-way absorption, $A_{R}$, for an emitter at sea level for $\theta>10^{\circ}$ is

$$
A_{R}=\frac{A_{R z}}{\sin \theta} \mathrm{dB}
$$

As in (17), for elevation angles of $\theta \geq 10^{\circ}, A_{R}$ is modified to account for the fact that the emitter is not at sea level by

$$
A_{R}=\frac{\gamma_{R} h_{R} e^{-h_{a c} / h_{R}}+\gamma_{w} h_{w} e^{-h_{a c} / h_{w}}}{\sin \theta} \mathrm{dB} .
$$

Similarly, as shown in (18), for elevation angles between $0^{\circ}$ and $10^{\circ}$,

$$
A_{a}=\frac{\sqrt{a_{e}}}{\cos \theta}\left[\gamma_{R} \sqrt{h_{R}} e^{-h_{a c} / h_{R}} \mathbf{F}\left(\tan \theta \sqrt{\frac{a_{e}}{h_{R}}}\right)\right] \mathrm{dB},
$$

where the function, $\mathbf{F}$, is the same as (19).

3.3. Summary of Atmospheric Effects. The two main atmospheric effects that affect ADS-B signals are ionospheric scintillation and gaseous absorption. As a result of known inaccuracies of the ITU-R scintillation model, its effect is not included in the analysis. Faraday rotation is not significant as the satellite will have a circular polarized antenna. Time delay is not important as the messages arrive at random times and the preamble of ADS-B messages contains synchronization bits. Lastly, the CanX-7 ADS-B receiver is a power detector that samples only eight times during the pulse length $(16 \mathrm{MHz}$ sample rate on a $0.5 \mu$ s pulse), so time delay dispersion and phase angle dispersion are not significant. Table 1 summarizes atmospheric effects discussed in this section.

\section{ADS-B Signal Propagation Model}

\subsection{Environmental Data}

4.1.1. Total Electron Content. Data for TEC values were sourced from the Centre for Orbit Determination in Europe $\mathrm{ftp}$ site that is operated by the Astronomical Institute of the University of Bern, Switzerland, Centre for Orbit Determination in Europe. Data were provided in an IONEX format where maps are calculated every two hours on even hours over a $2.5^{\circ}$ latitude by $5^{\circ}$ longitude grid and presented in tabular form. These maps were created using the single thin layer model (Section 3.1) at a height of $450 \mathrm{~km}$. Values at interstitial points were obtained by initially using bilinear interpolation from the four nearest grid points that surrounded the IPP for each map and then a linear interpolation in time between the two maps to the specific event time. 
TABLE 1: Atmospheric effects for an ADS-B signal with an elevation angle of $30^{\circ}$.

\begin{tabular}{lc}
\hline Effect & Value \\
\hline Faraday rotation & $12.5^{\circ}$ \\
Time delay & $26 \mathrm{~ns}$ \\
Time delay dispersion & $83 \mathrm{ps}$ \\
Phase dispersion & $16.3^{\circ}$ \\
Ionospheric scintillation & Up to $-20 \mathrm{~dB}$ at the geomagnetic \\
Ionospheric absorption & equator at equinox \\
Gaseous absorption & Negligible \\
Particulate & $-0.114 \mathrm{~dB}$ in rain for multipath \\
Tropospheric scintillation & case \\
\hline
\end{tabular}

4.1.2. Magnetic Field. There was the choice of two main magnetic models for use in this simulation: the World Magnetic Model (WMM) 2010 and the International Geomagnetic Reference Field (IGRF) 11. WMM 2010 is a model jointly developed by the UK and US defence departments and it is predominantly used by the North Atlantic Treaty Organization and the International Hydrographic Organization. IGRF11 is a standard mathematical description of the large-scale features of the Earth's magnetic field and it is predominantly used by the scientific community. As IGRF11 has greater recognition in the scientific community, it was selected for the model. A series of points corresponding to the TEC grid at an altitude of $450 \mathrm{~km}$ were programmed into the model and the resultant grid values were used to obtain specific magnetic field values at the IPP by bilinear interpolation.

4.2. North Atlantic Air Space. Oceanic airspace differs from most domestic airspace in that aircraft separation is achieved procedurally rather than using radar. To achieve this separation, a protected "box" of airspace exists that extends \pm 90 nautical miles along track and \pm 25 nautical miles across track centered on the aircraft's predicted position. ATS providers recommend a system of potential tracks provided by the NATS. Tracks vary daily according to prevailing wind conditions.

4.3. Aircraft. Aircraft position data were obtained from NAV CANADA for a 24-hour period on 29 April 2012 that described every aircraft transiting through Gander and Shanwick OCAs. Figure 3 shows the number of aircraft in these regions as a function of time of day. Two peaks are evident: the peak at about 0300 UTC representing the eastward flow of aircraft and the broader peak at 14001500 UTC representing the westward flow of aircraft. The maximum number of aircraft during these peaks is 218 and 216 , respectively.

4.3.1. ADS-B Equipment. A selection of ADS-B capable transponders was sourced from the International Civil Aviation Organization ADS-B Task Force document describing

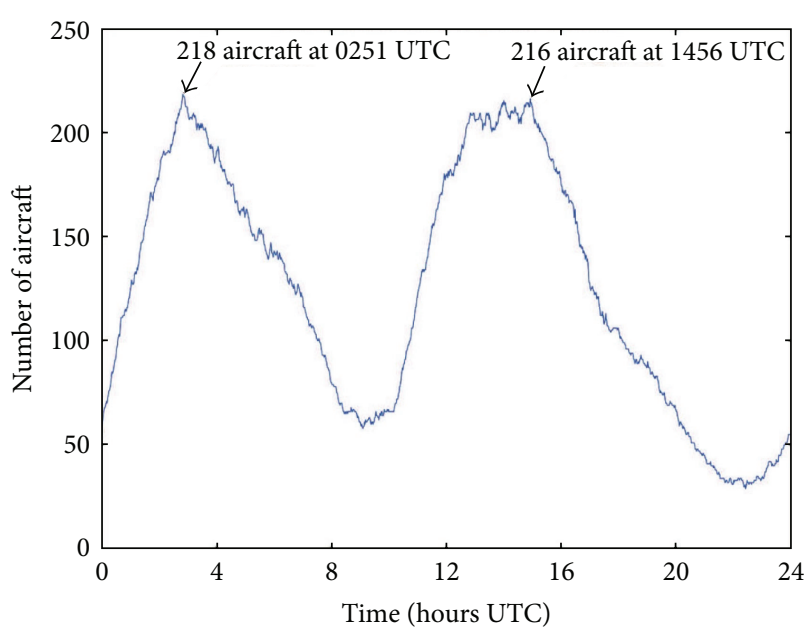

FIGURE 3: Aircraft traffic in Gander/Shanwick Oceanic Control Areas on 29 April 2012.

the availability from major avionics vendors. As the focus of this study is on large commercial aircraft transiting the North Atlantic region, only transponders for these aircraft were considered, while systems common on business and general aviation aircraft were omitted. The majority of ADS$\mathrm{B}$ enabled transponders used on large commercial aircraft can output more than the upper limit of $500 \mathrm{~W}$ mandated by the DO-260 [1]. This is to ensure that installation losses can be overcome so that a full $500 \mathrm{~W}$ output power is present at the antenna. Both Boeing and Airbus commercial fleets, as well as a large number of military aircraft, use the S655366 antenna made by Sensor Systems. Each aircraft has an antenna fitted to both the upper and the lower surfaces to achieve spherical coverage. Figure 4 shows the antenna radiation pattern provided by Sensor Systems, Inc., for the S65-5366 antenna at $1100 \mathrm{MHz}$. Figure 4(a) demonstrates that the antenna meets the specification of behaving like an ideal quarter-wave monopole coplanar to the aircraft [1]. Figures 4(b) and 4(c) show the longitudinal and lateral radiation patterns. To simplify the model, it was assumed that the lefthand side of the longitudinal pattern is rotated about the $z$ axis so that there is no azimuthal dependence.

4.4. Satellite Parameters. Satellite orbital geometry was determined with AGI's Satellite Toolkit (STK) using orbital altitudes of 400, 600 and $800 \mathrm{~km}$. These altitudes were chosen since the CanX-7 mission should fall within this range once a launch is brokered. The ADS-B receiver antenna is a circularly polarized microstrip patch antenna that possesses a wide beamwidth and corresponding low gain. Figure 5 shows the receiver footprint at an altitude of $800 \mathrm{~km}$, which has a width of approximately $2700 \mathrm{~km}$, during the middle of the pass used in the simulation.

4.5. ADS-B $1090 \mathrm{MHz}$ Signal. A number of aircraft systems transmit in the $1090 \mathrm{MHz}$ band, including Modes A, C, and $S$ transponders, Traffic Collision Avoidance System (TCAS), and ADS-B. As the area of interest over the centre of the North 


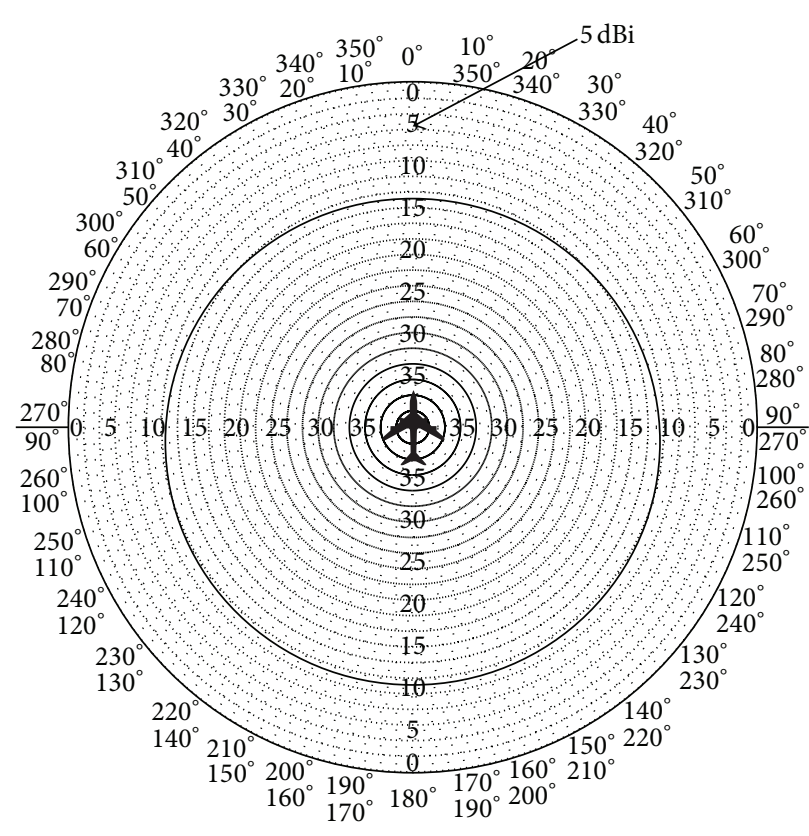

(a)

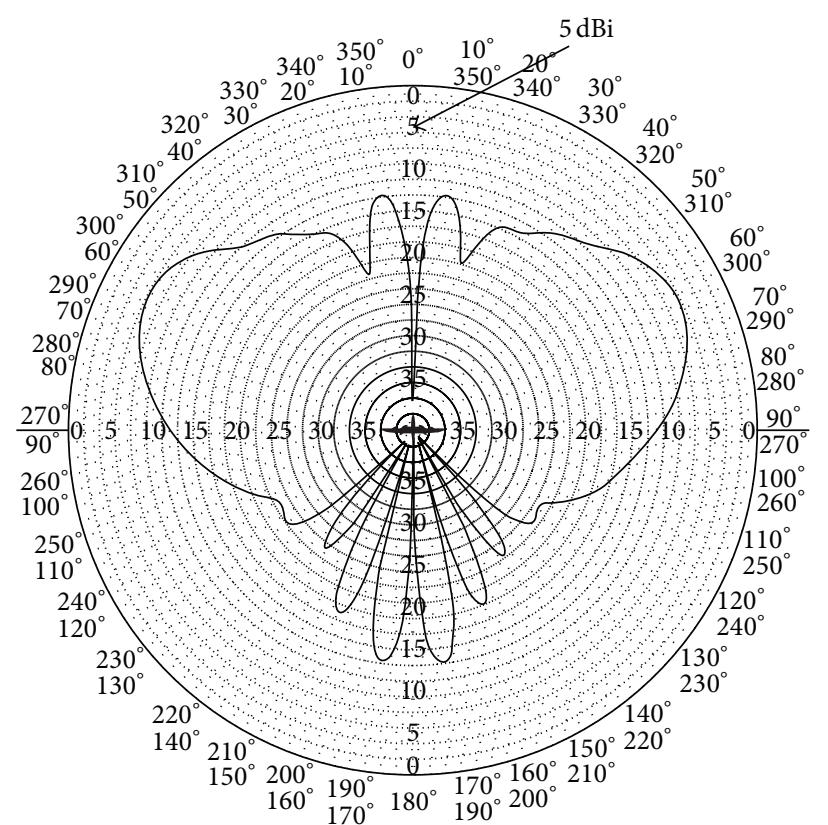

(b)

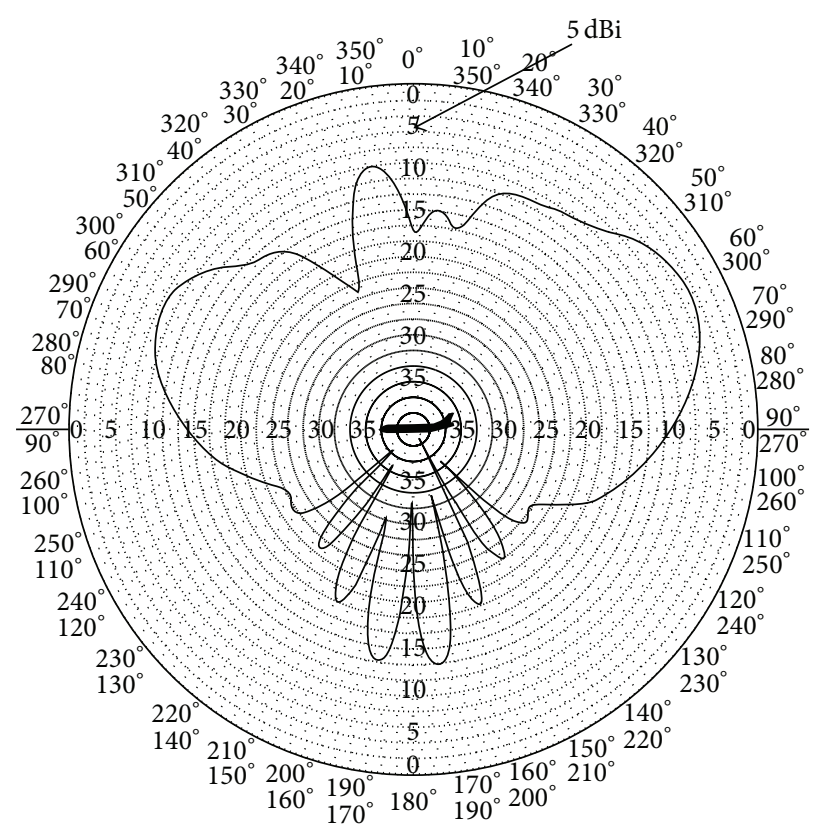

(c)

FIGURE 4: S65-5366 antenna radiation pattern at $1100 \mathrm{MHz}$ for (a) azimuth, (b) longitudinal, and (c) lateral directions (Sensor System, Inc.).

Atlantic region is out of range of secondary surveillance radars (SSRs), transponders normally do not transmit. The exception to this is described in TCAS operation. TCAS is a system that was designed to increase cockpit awareness of nearby aircraft and suggest avoidance manoeuvres if required. It achieves this by passively monitoring ADS-B messages or other aircraft transponder transmissions to track intruders that are not projected to be near-term collision threats. If required, TCAS can also actively interrogate aircraft that appear to be a collision threat. To aid monitoring, all
TCAS-equipped aircraft must broadcast a Mode S DF11 “All Call” message every 0.8 to $1.2 \mathrm{~s}$ [14]. A DF11 message is pulse position modulated (PPM) at a $1 \mathrm{MHz}$ rate. It is $64 \mu \mathrm{s}$ long, with an $8 \mu$ s preamble for synchronization and a $56 \mu$ s data block. ADS-B signals, also known as DF17 messages, are similarly modulated with the same $8 \mu$ s preamble but have a longer $112 \mu$ s data block. The structure of Mode S and ADS-B messages is shown in Figure 6.

The four main types of ADS-B messages convey position, velocity, event, and identification information. As the 


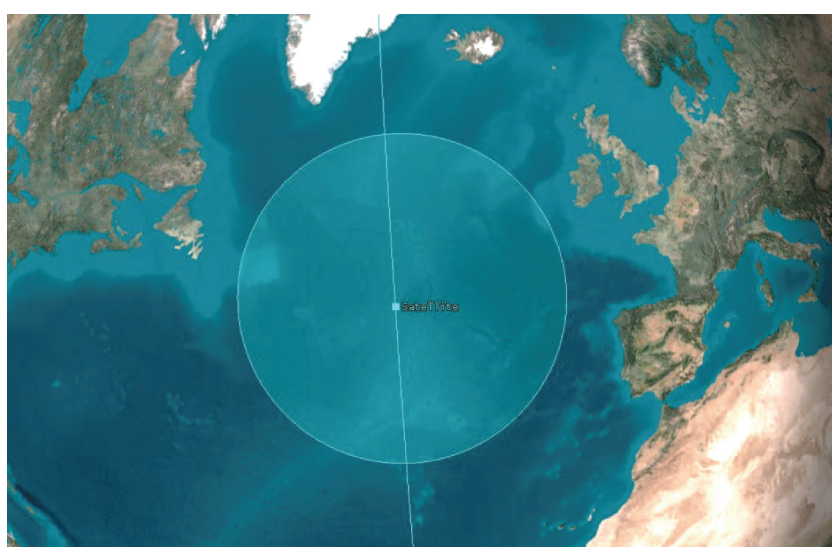

FIGURE 5: Receiver footprint for proposed CanX-7 ADS-B receiver (AGI STK Software).

TABLE 2: ADS-B message transmission intervals [14].

\begin{tabular}{lcc}
\hline Message type & Lower limit (s) & Upper limit (s) \\
\hline ADS-B position & 0.4 & 0.6 \\
ADS-B velocity & 0.4 & 0.6 \\
ADS-B event & 0.4 & 0.6 \\
ADS-B identification & 4.8 & 5.2 \\
\hline
\end{tabular}

messages are short, multiple messages have to be transmitted frequently according to the random intervals described in Table 2. This equates to an average of 6.2 messages per second, with successive messages of each type transmitted alternately from the top and bottom antennas.

4.6. Model Description. The model uses Python as the main programming language. Initially, various data files, including aircraft and satellite positions, environmental data, and antenna radiation patterns, were parsed from their native form into unique/specific database tables. Then, as it was desired to have the model operate at a $1 \mathrm{~Hz}$ rate, individual aircraft positions were interpolated from the NAV CANADAprovided data where typical periods between successive position reports range between 40 and 50 minutes. Satellite positional data were calculated at a $1 \mathrm{~Hz}$ rate from the scenario developed with AGI STK software. Relative geometry between the satellite and each aircraft position was calculated to yield all the required distances and angles in addition to the position of the IPP. The IPP position was then used to obtain TEC and magnetic field parameters. Path losses and contributions from antenna radiation patterns were calculated and received power at the satellite, $P_{r}$, was determined:

$$
P_{r}=\frac{P_{t} G_{t} G_{r} \lambda}{(4 \pi)^{2} R_{d}^{2} L_{a}},
$$

where $P_{t}$ is the transmitter power, $G_{t}$ and $G_{r}$ are transmitter and receiver gains, respectively, and include line losses, $\lambda$ is the wavelength of the $1090 \mathrm{MHz}$ transmission $(0.275 \mathrm{~m}), R_{d}$ is the direct path length from transmitter to satellite receiver, and $L_{a}$ is the atmospheric loss. Messages were generated according to the random transmission intervals shown in Table 2 and then sorted chronologically.

The modular nature of the model's architecture is one of its strengths and provides flexibility. For example, if the scenario was to compare the performance of two satellite antennas, it would be easy to load the respective performance data and conduct only the calculations required to determine the contribution of the antennas' radiation pattern. This could be done without having to regenerate all of the aircraftsatellite geometries.

It should be noted that the model also calculates the strength of ocean-reflected signals from the bottom antenna, based on aircraft geometry, sea surface roughness, and sea surface temperature. Reflected signals were below the sensitivity of the ADS-B receiver [15] and have not been included in this paper.

\section{Simulation Results}

A scenario was run to examine the power received at the input to the satellite receiver for the maximum permitted transmitter power of $500 \mathrm{~W}$ at the aircraft antenna at angles relative to the satellite's nadir. Satellite altitudes of 400, 600, and $800 \mathrm{~km}$ were input into the model. The two receiver sensitivity thresholds of -98.5 and $-103 \mathrm{dBm}$ were compared to the resultant signal power. The results are shown in Figure 7.

A significant observation is that all ADS-B signals between approximately $3^{\circ}$ and $60^{\circ}$ from nadir to aircraft are above the $-103 \mathrm{dBm}$ threshold for all altitudes, while a small portion of the signals is below the $-98.5 \mathrm{dBm}$ threshold for altitudes of 600 and $800 \mathrm{~km}$. Examination of the trace in Figure 7 for all altitudes reveals a circular null directly under the satellite. At $800 \mathrm{~km}$ altitude for a receiver sensitivity of $-103 \mathrm{dBm}$ this null is approximately $60 \mathrm{~km}$ in diameter. With an orbital velocity of approximately $7.5 \mathrm{~km} / \mathrm{s}$, an aircraft could potentially be in this null for a maximum of 10 seconds. Additionally, there is an annulus between $10^{\circ}$ and $20^{\circ}$ where the signals are weaker. At $800 \mathrm{~km}$ altitude for a receiver sensitivity of $-103 \mathrm{dBm}$ the annulus is $30 \mathrm{~km}$ wide centred at $215 \mathrm{~km}$ radius from the satellite nadir (Figure 8 ). If the receiver sensitivity is $-98.5 \mathrm{dBm}$, the null at nadir increases to $85 \mathrm{~km}$ diameter and the annulus increases to a width of $180 \mathrm{~km}$ centred at a radius of $245 \mathrm{~km}$ from the satellite nadir (Figure 8 ). The extent of both the null and annulus would be reduced for lower altitudes. The cause of the null and the annulus is a function of the aircraft antenna radiation pattern. The null is typical of a quarter-wave monopole (Figure 4(a)), while the annulus is related to the lateral radiation pattern (Figure 4(c)).

\section{Conclusion}

A model was created to assess the performance of an ADS-B receiver scheduled to be launched on the CanX-7 nanosatellite. The model takes into account neutral atmosphere and ionospheric effects, aircraft-satellite geometry, and antenna 


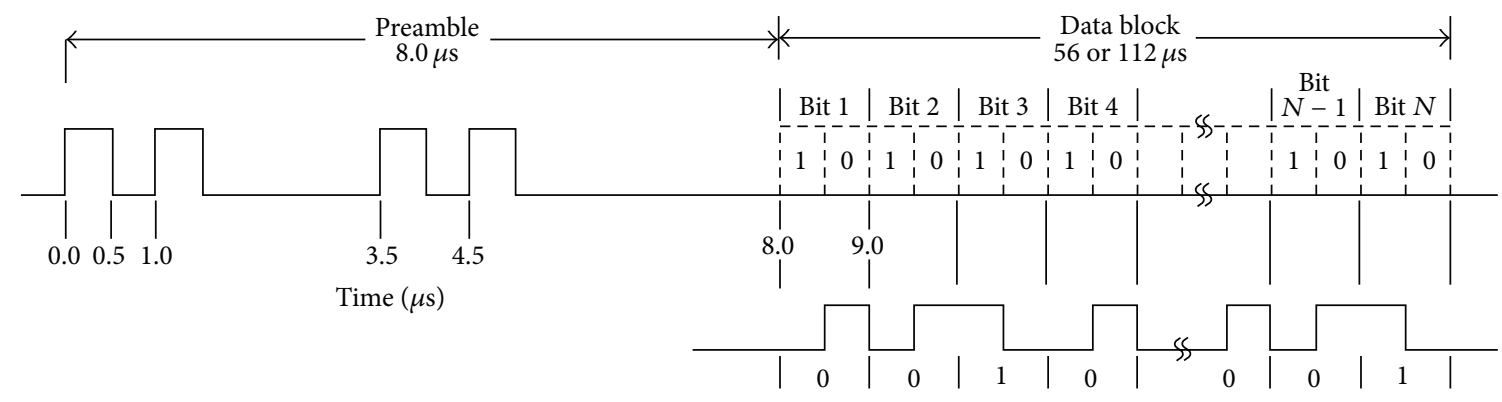

FIGURE 6: ADS-B and Mode S reply waveform [14].
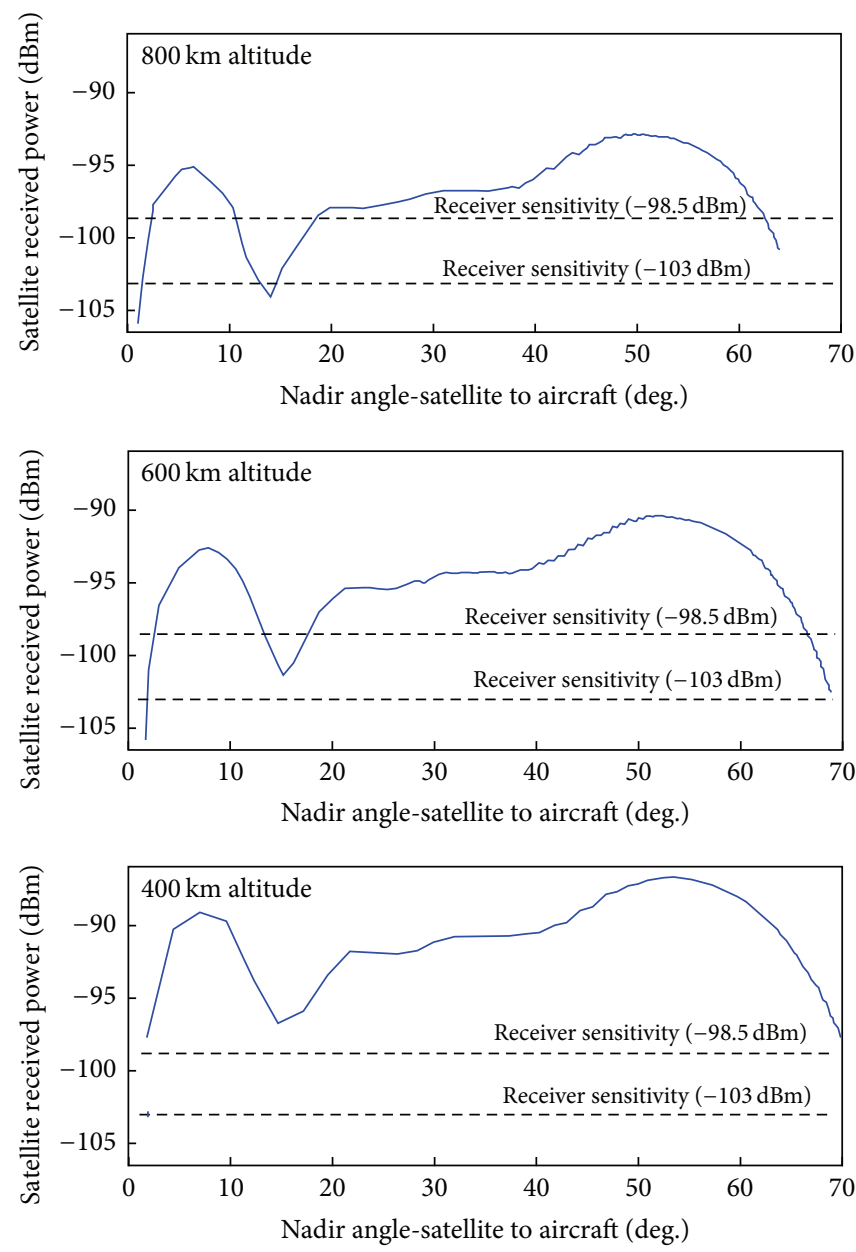

FIGURE 7: ADS-B signal strength observed at satellite at 400, 600 and $800 \mathrm{~km}$ altitude.

radiation patterns. The region of interest for this study is the North Atlantic region as this area presently uses procedural air traffic services instead of radar-based services that are used over land. It is also relatively quiet in the $1090 \mathrm{MHz}$ frequency band.

A simulation was run by inserting real aircraft data from the NATS into the model and placing the satellite in LEO. The simulation examined the power received at the satellite for direct path cases over a range of satellite to aircraft geometries at altitudes of 400, 600, and $800 \mathrm{~km}$.
The key findings of the simulation are as follows:

(i) The ADS-B antenna/receiver proposed for CanX-7 is viable for altitudes between 400 and $800 \mathrm{~km}$.

(ii) Discounting the effect of the aircraft antenna radiation pattern restrictions, almost all direct path signals are detectable with a wide-beamwidth, low gain antenna.

(iii) There is a null at nadir in which there could be no detection for a maximum of 10 seconds. 

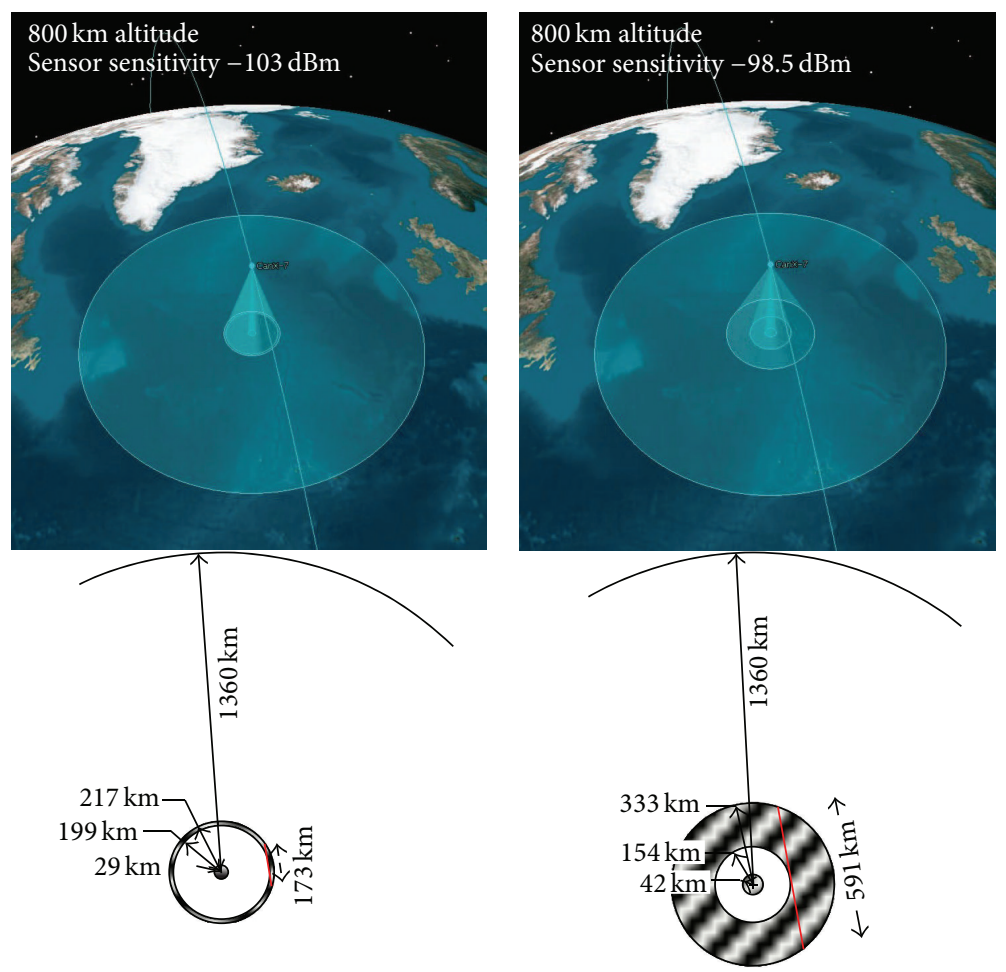

FIGURE 8: ADS-B receiver coverage for -103 and $-98.6 \mathrm{dBm}$ receiver sensitivity at $800 \mathrm{~km}$ (AGI STK Software).

Future work is divided into refinement and validation of the model. The first refinement is to remove the simplification of azimuth independence. Antenna radiation patterns would need to include azimuth information. Faraday rotation calculations would then be required to determine the plane of polarization at the satellite antenna. The second refinement is to incorporate ionospheric scintillation effects. Even though the ITU-R scintillation model is known to be inaccurate, its inclusion would provide a rough estimate of the effect on signal throughput. The fourth and most complex model refinement is to include the effect of SSR and TCAS signals around the domestic/oceanic airspace boundary. Signal density in this region is higher than in the mid-Atlantic as ground-based SSRs interrogate aircraft that reply with Mode A, C, or S messages as appropriate. Detailed knowledge of these radars such as scan rates, interrogation patterns, and mutual interference reduction plans would be required in addition to the increased data of aircraft movements. TCAS transmissions are also expected to be greater as aircraft are in closer proximity on airways and near airports.

Validation of the model will be possible with payload data from the CanX-7 satellite. Aircraft positional information would have to be sourced from NAV CANADA to provide truth data. Once the model has been validated and refined, it could be employed in simulating follow-on ADS-B receiver systems and future air traffic scenarios.

\section{Conflict of Interests}

The authors declare that there is no conflict of interests regarding the publication of this paper.

\section{References}

[1] RTCA DO-260B, Minimum Operational Performance Standards for $1090 \mathrm{MHz}$ Extended Squitter Automatic Dependent Surveillance-Broadcast (ADS-B) and Traffic Information Services-Broadcast (TIS-B), Radio Technical Commission for Aeronautics, 2009.

[2] O. P. Gupta, "Global augmentation of ADS-B using Iridium NEXT hosted payloads," in Proceedings of the 11th Integrated Communications, Navigation and Surveillance Conference (ICNS '11), pp. 1-15, May 2011.

[3] R. Francis, R. Vincent, J.-M. Noël et al., "The flying laboratory for the observation of ADS-B signals," International Journal of Navigation and Observation, vol. 2011, Article ID 973656, 5 pages, 2011.

[4] A. C. Cushley and J.-M. Noël, "Ionospheric tomography using ADS-B signals," Radio Science, vol. 49, no. 7, pp. 549-563, 2014.

[5] R. Francis, J. Noël, and R. Vincent, "Orbital monitoring of automatic dependent surveillance-broadcast (ADS-B) signals for improved air traffic surveillance in remote and oceanic airspace," in Proceedings of the 62nd International Astronautical Congress, University of Western Ontario, Cape Town, South Africa, October 2011.

[6] R. Van Der Pryt and R. Vincent, "A Simulation of signal collisions over the North Atlantic for a spaceborne ADS-B receiver using Aloha protocol," Positioning, vol. 6, no. 3, pp. 2331, 2015.

[7] G. Bonin, J. Hiemstra, T. Sears, and R. E. Zee, "The CanX7 drag sail demonstration mission: enabling environmental stewardship for nano- and microsatellites," in Proceedings of the AIAA/USU Conference on Small Satellites, Technical Session XI: Around the Corner, SSC13-XI-9, August 2013. 
[8] L. Ippolito, Propagation Effects Handbook for Satellite System Design-Section 2 Prediction, Revision 01, ACS Stanford Telecom, 5th edition, 1999, Prepared for the NASA Jet Propulsion Laboratory.

[9] D. A. Smith, E. A. Araujo-Pradere, C. Minter, and T. FullerRowell, "A comprehensive evaluation of the errors inherent in the use of a two-dimensional shell for modeling the ionosphere," Radio Science, vol. 43, no. 6, 2008.

[10] J. E. Allnutt, Ed., Satellite-to-Ground Radiowave Propagation: Theory, Practice and System Impact at Frequencies above $1 \mathrm{GHz}$, Peregrinus on Behalf of the IEEE, 1989.

[11] ITU-R P.531-12, "Ionospheric Propagation Data and Prediction Methods Required for the Design of Satellite Services and Systems," International Telecommunication UnionRadiocommunication Sector, Study Group 3, 2013.

[12] ITU-R P.838-3, Specific Attenuation Model for Rain for Use in Prediction Method, Study Group 3, International Telecommunication Union-Radiocommunication Sector, 2005.

[13] ITU, "Rain height model for prediction method," ITUR P.839-2, International Telecommunication Union-Radiocommunication Sector, Study Group 3, 1999.

[14] RTCA DO-181D, Minimum Operational Performance Standards for Air Traffic Control Radar Beacon System/Mode Select (ATCRBS/Mode S) Airborne Equipment, Radio Technical Commission for Aeronautics, 2008.

[15] R. Van Der Pryt, Modelling Automatic Dependent SurveillanceBroadcast (ADS-B) signals received by a low-earth-orbiting satellite: examining the North Atlantic oceanic airspace control areas [M.S. thesis], Royal Military College of Canada, Kingston, Canada, 2014. 

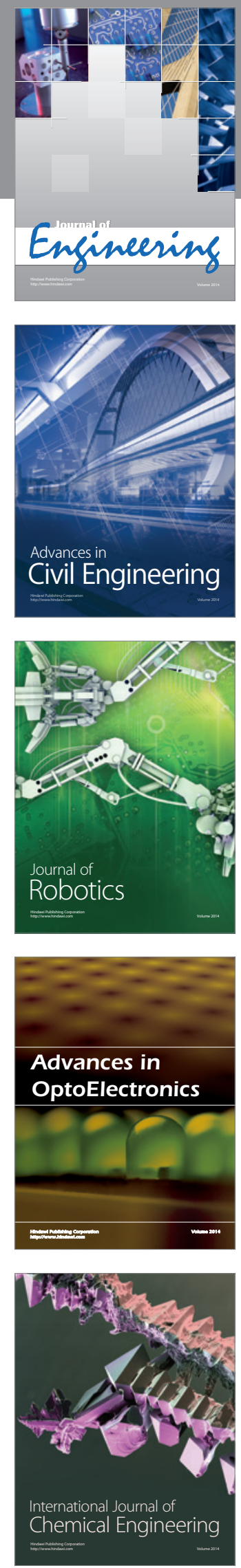

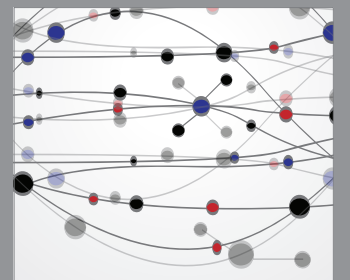

The Scientific World Journal
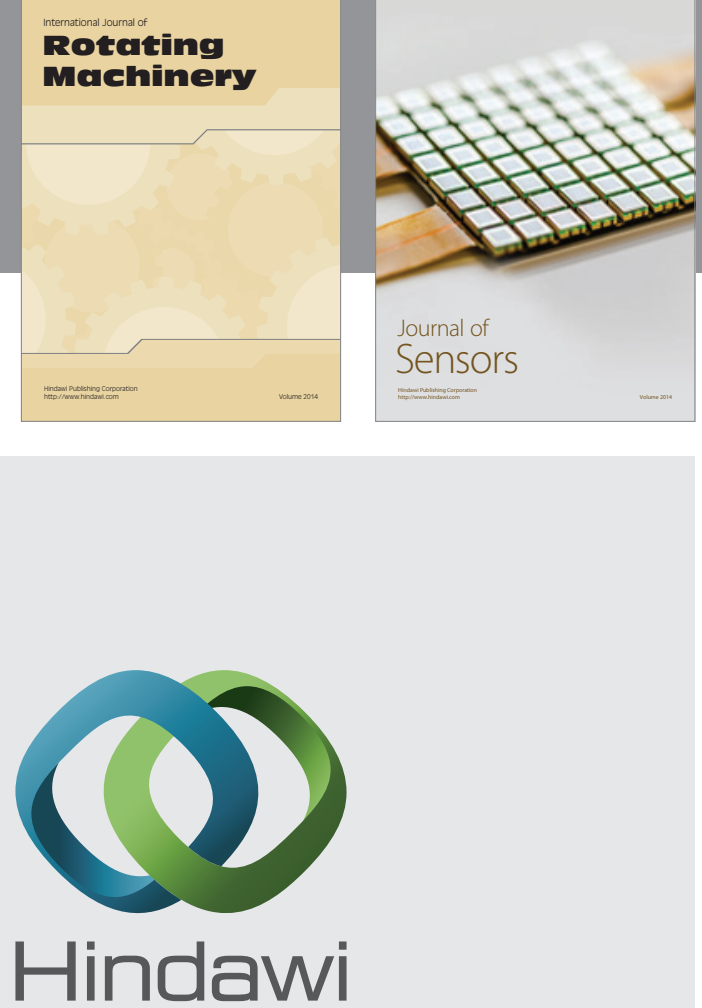

Submit your manuscripts at http://www.hindawi.com
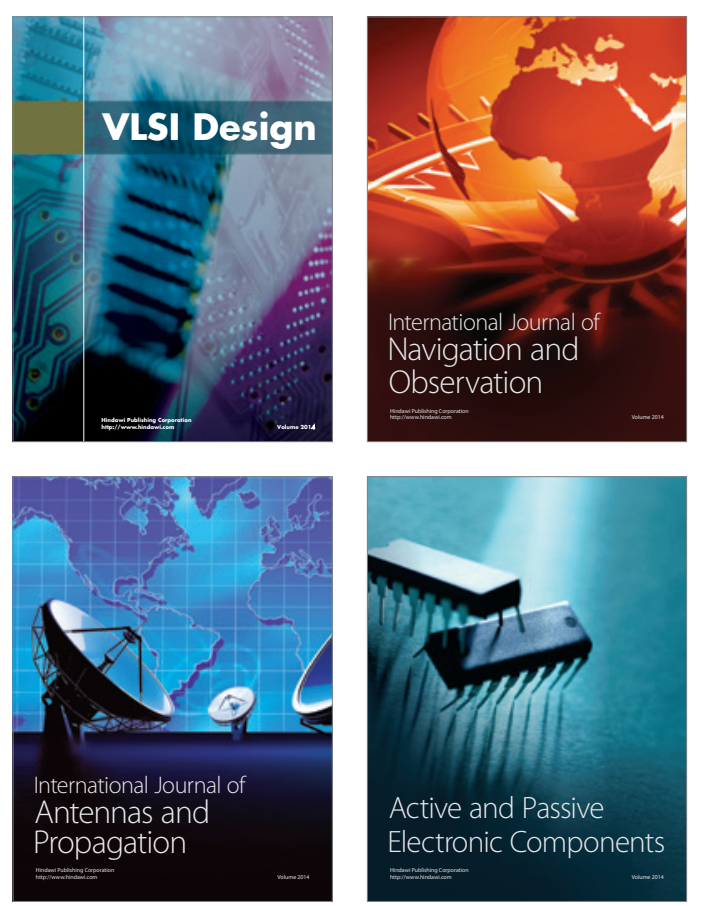
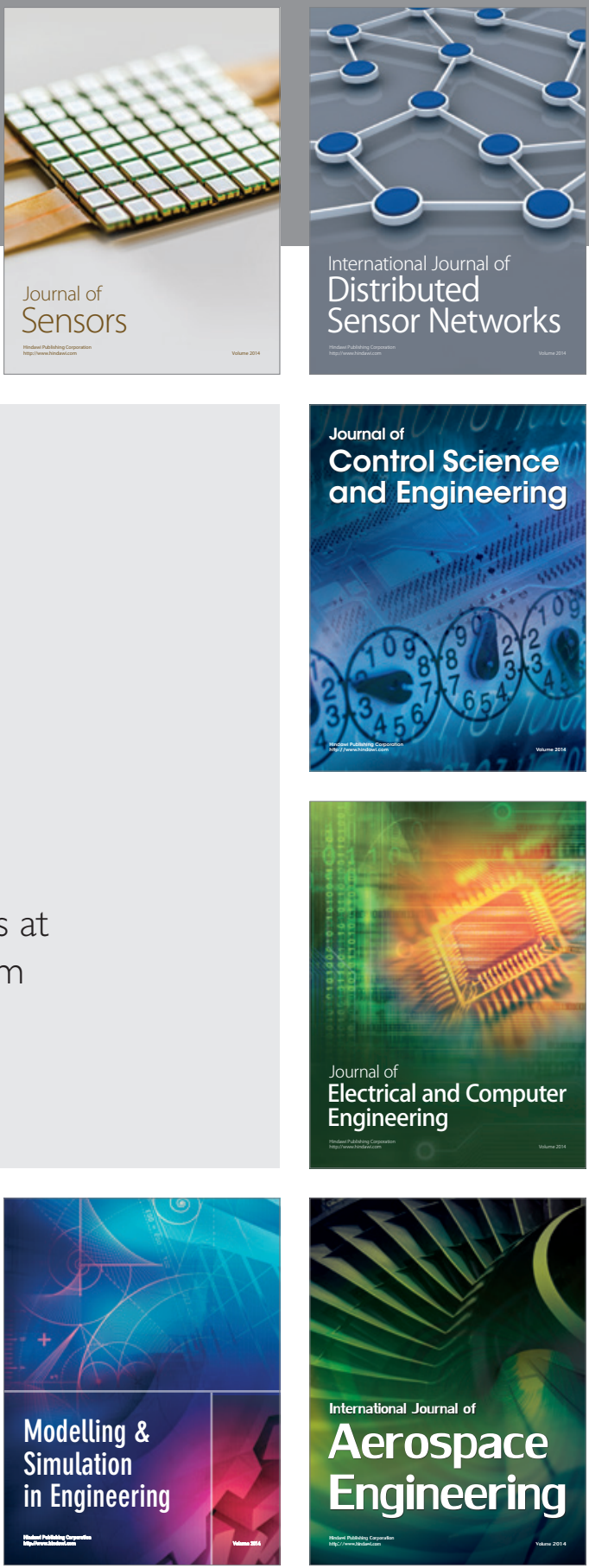

Journal of

Control Science

and Engineering
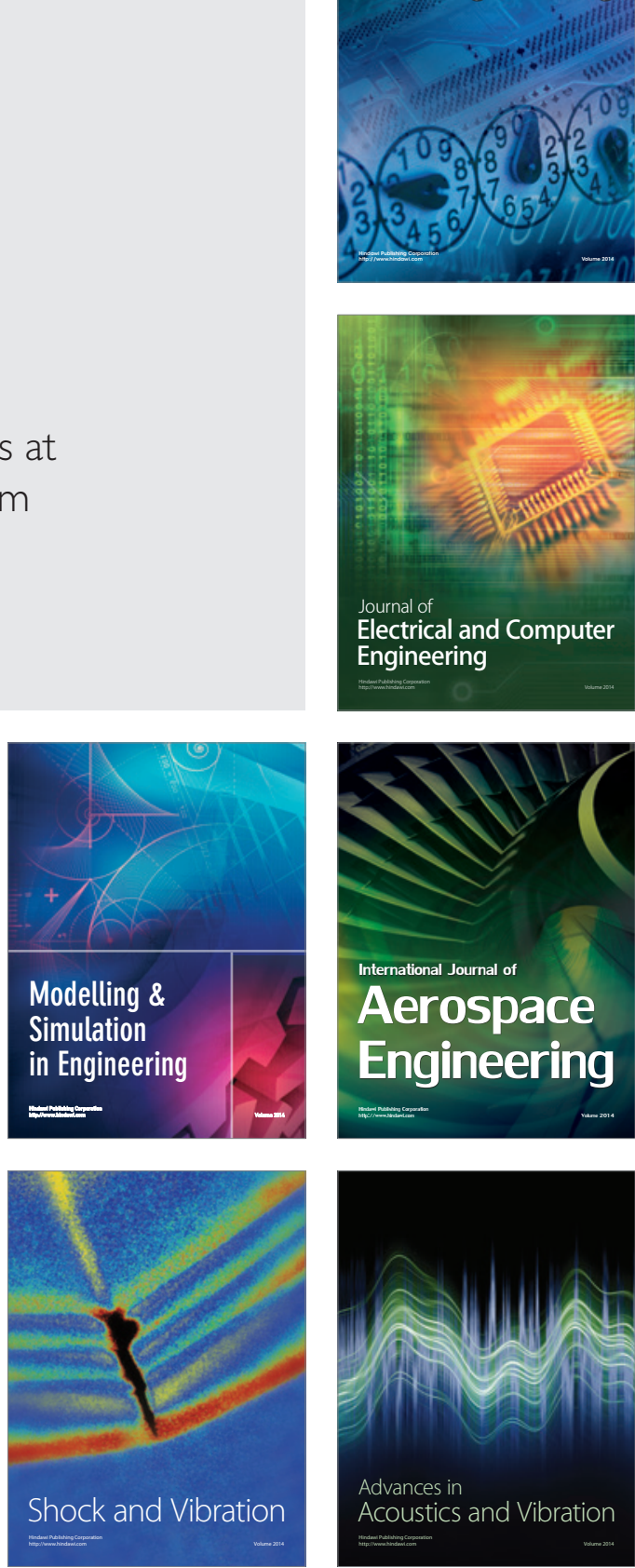